\title{
Music Therapy Intervention for the Mother- Preterm Infant Dyad: Evidence From a Case Study in a Brazilian NICU
}

\author{
Ambra Palazzi ${ }^{1}{ }^{*}$, Rita Meschini ${ }^{2}$, Cesar A. Piccinini ${ }^{1}$ \\ 1 Institute of Psychology, Federal University of Rio Grande do Sul, Brazil \\ 2 S. Stefano Rehabilitation Institute, Italy \\ *palazziambra@gmail.com
}

Received: 22 February 2017 ; Accepted: 31 May 2017 ; Published: 1 July 2017

Editor: Helen Oosthuizen Reviewers: Debbie Bates, Claire Ghetti

\begin{abstract}
This study investigated the contributions of music therapy to the mother-preterm infant dyad in a NICU. A single case study was carried out involving a mother and her preterm daughter $(27 \mathrm{GW})$. They participated in nine sessions of the Music Therapy Intervention for the Mother and her Preterm Infant -MUSIP, with the aim of sensitizing the mother to the importance of singing. After the MUSIP and in the preand post-discharge periods, the mother was interviewed in order to provide an assessment of the intervention; furthermore, the dyad was videotaped during singing and non-singing interactions. The interviews with the mother and the written descriptions of the video footage were examined through thematic analysis. The results showed music therapy contributed: a) to the empowerment of the infant, through relaxation, stabilization of oxygen saturation, the presentation of new competences and engagement in singing; and b) to the empowerment of the mother, through relaxation, the overcoming of embarrassment and the fear of interacting with the infant, and through the strengthening of her maternal competences and her autonomy in singing. Together, the results highlighted the importance of music therapy to the mother-infant interaction, because singing contributed to more prolonged face-toface contact and more diversified expressions of affection.
\end{abstract}

Keywords: Prematurity, NICU, Music therapy, Maternal singing, Mother-infant interaction

\section{Introduction}

Almost $11 \%$ of newborns worldwide are born before the 37th week of gestational age (WHO, 2010). Premature birth can affect the infant's long term development, and the hospitalization of the infant in the Neonatal Intensive Care Unit (NICU) is a traumatic experience for the whole family, as both mothers and fathers may experience higher levels of stress, anxiety, and depression (Flacking, Ewald, Nyqvist, \& Starrin, 2006; Fleck \& Piccinini, 2013; March of Dimes, PMNCH, Save the Children, \& WHO, 2012; Shaw et al., 2014).

The impact of prematurity on the mother-infant relationship is still a controversial issue. The recent meta-analysis conducted by Bilgin and Wolke (2015), who examined 
34 studies published between 1980 and 2013, showed no significant differences in the sensitivity and the responsiveness of mothers of term and preterm infants during their interaction in the first years of life. Nevertheless, some studies showed that prematurity might affect bonding and mother-infant interaction (Forcada-Guex, Pierrehumbert, Borghini, Moessinger, \& Muller-Nix, 2006; Korja, Latva, \& Lehtonen, 2012). Beyond the biological vulnerability involved in prematurity, adverse psychosocial factors represent a multiple-risk situation and require the implementation of early interventions (Linhares, Carvalho, Machado, \& Martinez, 2003; Moreira et al., 2011; White-Traut \& Norr, 2009).

Music therapy is an emergent discipline in this context, both in research and clinical work, and it showed positive effects on the infants, on their parents and on the parentinfant relationship (Haslbeck, 2012; Standley, 2012). Several studies highlighted the fetus's and newborn's early auditory abilities of recognizing and reacting to maternal voice and music (Al-Qahtani, 2005; Kisilevsky et al., 2003; McMahon, Wintermark, \& Lahav, 2012; Moon \& Fifer, 2000). Besides this, the mother-infant dialogue, defined by Malloch and Trevarthen (2009) as communicative musicality, is made of specific musical elements that contribute to the regulation of the infant's attention, the development of linguistic structure, the communication of emotions, and the regulation of social behavior (Butler et al., 2014). In particular, by emphasizing the musical elements naturally presented in infant-directed speech, maternal singing is especially powerful in enhancing mother-infant bonding (Peretz, 2010; Trehub, Becker, \& Morley, 2015).

According to the recent review of literature conducted by Palazzi, Nunes, and Piccinini (2017), music therapy interventions carried out by music therapists can be found among the music-based interventions in the NICU (Ettenberger et al., 2014; Haslbeck, 2014; Loewy, Stewart, Dassler, Telsey, \& Homel, 2013; Standley et al., 2010; Ullsten, Eriksson, Klässbo, \& Volgsten, 2016), as well as musical stimulation interventions conducted by other healthcare professionals (Alipour, Eskandari, Ahmari Tehran, Eshagh Hossaini, \& Sangi, 2013; Keidar, Mandel, Mimouni, \& Lubetzky, 2014) or by parents (Arnon et al., 2014; Filippa, Devouche, Arioni, Imberty, \& Gratier, 2013; Nöcker-Ribaupierre, Linderkamp, \& Riegel, 2015). These interventions can employ a receptive approach with recorded sounds and music (Alipour et al., 2013; Standley et al., 2010), or an active approach through live music and singing (Ettenberger et al., 2014; Haslbeck, 2014; Loewy et al., 2013; Malloch et al., 2012; Ullsten et al., 2016).

Music therapy and musical stimulation have shown positive effects on the preterm infant, on oxygen saturation, on the regulation of the heart rate, on respiratory rate, on sleep patterns, on non-nutritional sucking, on weight gain and on the reduction of the duration of hospitalization (Bieleninik, Ghetti, \& Gold, 2016; Haslbeck, 2012; Standley, 2012). Among the music therapy interventions, we highlight those that employ live contingent or infant-directed singing with lullabies, parents' preferred songs and improvised singing (Malloch et al., 2012; Shoemark, 2011; Haslbeck, 2014; Loewy, 2015; Ullsten et al., 2016). For example, Haslbeck (2014) investigated the interactive potential of Creative Music Therapy (CMT, Nordoff \& Robbins, 1977) on 18 preterm infants. The author used an inductive approach based on the principles of grounded theory (Glaser \& Strauss, 1967; Strauss \& Corbin, 1998) and therapeutic narrative analysis (Aldridge \& Aldridge, 2002). By analyzing the video footage of the intervention and parental interviews, the author identified several categories, such as communicative musicality from episodes of interactional synchrony between the music therapist and the infant, the music therapist's responsiveness to the infant's behaviors, and the infant's and parents' empowerment. According to Haslbeck (2013, 2014), through communicative musicality in the CMT, both parents and infants might be empowered to self-regulate, orientate, participate, and engage in the interaction between them. Results suggested that CMT enhances self-regulation, orientation, and a greater engagement on the part of the infant, as well as promoting parental sensitivity.

Besides supporting the preterm infant's development, music-based interventions promote maternal well-being, by reducing stress and anxiety (Ak, Lakshmanagowda, G C M, \& Goturu, 2015; Arnon et al., 2014; Bieleninik et al., 2016; Cevasco, 2008) and by 
enhancing breastfeeding (Ak et al., 2015; Keith, Weaver, \& Vogel, 2012; Vianna et al., 2011). In particular, interventions that involve maternal singing and speech have positive effects on both the infant and the mother (Arnon et al., 2014; Filippa et al., 2013). Finally, some studies showed that music therapy contributes to the mother-infant interaction by enhancing attachment, parental responsiveness, interactional synchrony and communicative musicality between mother and infant (Cevasco, 2008; Ettenberger et al., 2014; Haslbeck, 2014; Walworth, 2007).

Research showed greater benefits of music therapy with live music (Arnon et al., 2006; Garunkstiene, Buinauskiene, Uloziene, \& Markuniene, 2014) and emphasized the importance of the parent's inclusion and accompaniment (Ettenberger et al. 2014; Edwards, 2011; Haslbeck, 2014). Nevertheless, most research focuses on the effects of recorded music on the preterm infant, without investigating maternal perspectives and mother-infant interaction (Haslbeck, 2012). Therefore, the aim of this study was to investigate the contributions of music therapy to the mother-preterm infant dyad in a Brazilian NICU, based on the structure of themes and on the theoretical background of Haslbeck's studies $(2013,2014)$. Our initial expectation was that music therapy would contribute to help both the mother and the infant to relax, to sensitize the mother to the importance of singing in the NICU as a resource for interacting with the infant, and to enhance the quality of mother-infant interaction.

\section{Methods}

\section{Participants}

Participants were a mother (Natalia) ${ }^{1}$ and her preterm daughter (Ana), who had no syndromes or congenital anomalies and was admitted to the NICU of a public hospital in Porto Alegre (State of Rio Grande do Sul, Brazil).

Natalia was 24 years old; she lived in Porto Alegre, had concluded primary education, and was unemployed. She had three children: two of them, 1 and 3 years old, were from previous relationships, whilst Ana was the daughter of her third partner who had been incarcerated almost a year earlier. The family had a low socioeconomic income. Ana was born at 27 weeks $^{2}$ of gestational age with a weight of $685 \mathrm{~g}$. The Apgar Score $^{3}$ at birth was 2 (1st minute), 4 (5th minute), and 6 (10th minute). After birth, due to symptoms of sepsis and respiratory distress, Ana was put in an incubator and submitted to orotracheal intubation. Besides the preterm birth, the infant presented no other severe clinical condition. After 118 days of hospitalization in the NICU, Ana had a weight of $2620 \mathrm{~g}$ at discharge; she could breathe autonomously and breastfeed.

\section{Design, procedures and instruments}

A single case study design (Stake, 2006) was used, with five phases of data collection. In Phase 1 (Pre-intervention), 20 days after the infant's birth, the mother was invited to participate in the study, she signed the Informed Consent Form and took part in the following interviews: the Interview about motherhood in the context of prematurity (NUDIF/PREPAR, 2009a), which was used to investigate the experience of motherhood in the post-partum period; and the Interview about the mother's musical history (Palazzi, Meschini, \& Piccinini, 2014a), which investigated the acoustic environment and the mother's experiences and musical preferences throughout life and during pregnancy. Both interviews were structured but conducted in a semi-directed manner. Moreover, a Demographic data sheet (NUDIF/PREPAR, 2009b) was filled out in this phase together with a Clinical data sheet for the mother and her preterm infant/post-partum (NUDIF/ PREPAR, 2009c), which was updated at each phase of data collection.

In Phase 2 (Intervention), a week after Phase 1, the mother participated in the Music Therapy Intervention for the Mother-Preterm Infant Dyad - MUSIP (Palazzi, Meschini, \& Piccinini, 2014b). The MUSIP is a music therapy intervention that aims at sensitizing the mother to the importance of singing to her infant and supporting the mother-infant dyad during maternal singing. It was created for this study based on music therapy 
research on prematurity. The MUSIP is organized in eight sessions, divided between meetings with the mother and sessions with the mother-infant dyad in the NICU. However, the structure of the MUSIP had to be adapted in this study in order to meet the mother's needs and the space availability in the NICU as described below. Besides this, due to Ana's clinical instability, the sessions with her started at 31 weeks of post-menstrual age ${ }^{4}$.

Session one only involved the mother, was conducted in a room of the Neonatology Unit and lasted approximately 45 minutes; sessions two, three, four, and five were carried out in the NICU with the mother and the infant in the incubator; furthermore, sessions six, seven, eight, and nine were held with Natalia during skin-to-skin contact with Ana (kangaroo care). The sessions with the dyad in the NICU lasted 15 to 20 minutes. In session one, the mother and the music therapist sang some songs selected by the mother ("Fico assim sem você" ["I stay like this without you"] by Claudinho and Buchecha and the Brazilian lullaby "Nana nenê" ["Rockabye baby"]), with and without guitar accompaniment. Sessions two, three and four aimed at accompanying and supporting the mother during the experience of singing for the daughter, by stimulating the observation of the infant's responses and by emphasizing the interactive potential of singing. Sessions five and six focused on the improvised humming with the dyad in the NICU and lastly, sessions seven, eight, and nine aimed at encouraging the mother to write a song for the infant. ${ }^{5}$ A music therapist, the first author of this study, conducted the nine sessions of the MUSIP (approximately once a week for 2 months) with the clinical supervision of another music therapist, the second author of this study.

In Phase 3 (Post-intervention), a week after the end of the MUSIP, the mother took part in the Assessment interview of the music therapy intervention for the mother and her preterm infant (NUDIF/PREPAR, 2014b), which investigated the impact of the intervention on maternal feelings about the music therapy, the therapist and her satisfaction with the intervention, as well as her perceptions about the changes in the infant and in their bonding. It was a structured interview conducted in a semi-directed manner.

Furthermore, the dyad participated in a session of the Observation of the motherpreterm infant interaction (Palazzi \& Piccinini, 2014), during maternal singing in the NICU. This session aimed at observing infant's and mother's behaviors and their interactions, during singing and non-singing episodes. The mother was oriented to interact with the infant approximately 2 to 8 minutes during each episode. The music therapist, with the help of a psychology student, videotaped the observation sessions by using a GoPro Hero 3+ camera within the NICU, and a Sony DCR-sr85 camera out of the NICU. The video footage focused on mother-infant interaction.

In Phase 4 (Pre-discharge), carried out before the infant's discharge, the mother took part in another assessment interview adapted for the pre-discharge phase, and a new observation session was conducted during breastfeeding in the NICU. To conclude, in Phase 5 (Post-discharge), a week after discharge, a new observation session was held in a hospital room in two different moments: during maternal singing and during nonsinging while changing the diaper. The first author carried out all the MUSIP sessions, the observation sessions in Phase 3 and 5, and all maternal interviews in Phase 1. A psychology student conducted the observation session in Phase 4, as well as all the clinical data sheets of the infant throughout the phases, and conducted the assessment interviews with the mother in Phase 3 and 4. All the observation sessions and the MUSIP sessions (with the exception of session two) were audio or videotaped. Two psychology students were responsible for transcribing maternal interviews. This study was approved by the Ethics Committees of the Psychology Institute of the Federal University of Rio Grande do Sul (UFRGS) (n. 985.941) and of the hospital (n. 1.069.283).

\section{Data analysis}

To analyze data, we examined the mother's interviews in the pre-MUSIP (Phase 1), post-MUSIP (Phase 3) and in the pre-discharge (Phase 4) period, as well as the video footage of the mother-infant dyad during MUSIP (Phase 2) and during the observation 


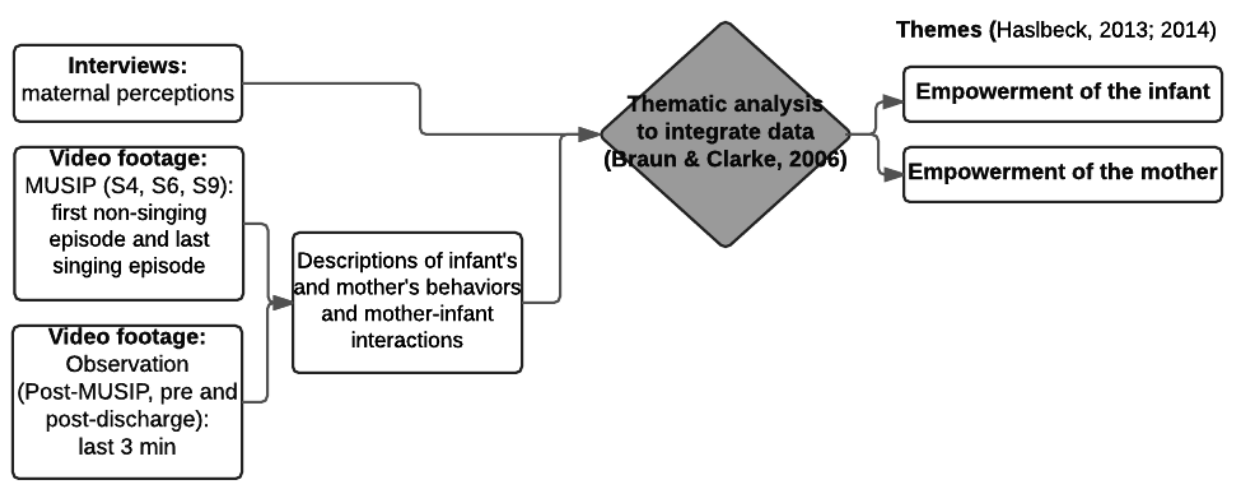

Figure 1. Flowchart of the analysis process.

sessions (Phase 3, 4, and 5). Initial interviews were used to describe the case. The assessment interviews in the post-MUSIP and in the pre-discharge period were examined through thematic analysis (Braun \& Clarke, 2006), with the aim of investigating maternal perceptions about the music therapy contributions to the mother-preterm infant dyad in the NICU, in particular to the infant, the mother and the mother-infant interaction.

Video footage of MUSIP sessions were analyzed as follows: 1. First, we watched the video footage and wrote a general report on all sessions; 2 . Secondly, we selected three of the six videotaped sessions (sessions four, six, and nine) ${ }^{6}$, and we identified and selected the first non-singing episode (3-4 minutes) and the last maternal singing episode (3-8 minutes) of each session, with a total of approximately 14 minutes of singing and 11 minutes of non-singing ${ }^{7} ; 3$. Lastly, the first author wrote detailed descriptions regarding infant's and mother's behaviors and mother-infant interactions during singing and non-singing episodes. The initial and final time of each behavior under observation were noted, so as to also have recorded information about the duration of infant's and mother's behaviors and their interactions.

We used a similar procedure for the video footage concerning the sessions of the $\mathrm{Ob}$ servation of the mother-preterm infant interaction (Phase 3, 4, and 5). 1. First, we identified and selected the last 3 minutes of each video excerpt, with a total of approximately 6 minutes of singing and 4 minutes of non-singing ${ }^{8} ; 2$. Secondly, the first author wrote detailed descriptions regarding infant's and mother's behaviors and mother-infant interactions during singing and non-singing episodes, highlighting the initial and final time of each behavior under observation, so as to have information about the duration of infant's and mother's behaviors and their interactions. A psychology student subsequently reviewed all video excerpt descriptions in an attempt to ensure consensus. Interviews and video excerpts contributed to data triangulation (Stake, 2006) allowing for an in-depth analysis of the case, based on maternal perceptions, on infant's and mother's behaviors, and on their interactions.

Data were integrated and analyzed through thematic analysis (Braun \& Clarke, 2006), using a deductive approach based on the structure of themes and on the theoretical background of Haslbeck (2013, 2014). The analysis was based on two themes: a) empowerment of the infant and b) empowerment of the mother. In particular, the interviews and the descriptions were read several times, so as to identify vignettes and excerpts of the descriptions that portrayed the themes used for analysis.

Haslbeck's $(2013,2014)$ structure of themes was originally created from studies that involved the therapeutic process between the music therapist and the infant. Therefore, this structure was adapted for the purposes of this study in order to consider the interactional process between mother and preterm infant. The original themes that referred to the music therapist's behaviors were adapted and employed to analyze maternal behaviors. The original structure also involved other themes, such as the responsiveness between the music therapist and the infant, which was not used in this study, 
since this category and all the dimensions about mother-infant interactions were included in the themes of empowerment of the infant and empowerment of the mother. Furthermore, the original structure involved the theme of communicative musicality, which was also investigated in this study, but it will be presented in a future publication. In conclusion, we added other characteristics to the themes based on the literature being specifically derived from data from this study.

\section{Results}

Results will be presented in two sections; the first one is related to the interviews and the observations about the empowerment of the infant, while the second one refers to the empowerment of the mother. During the presentation of the results, each theme will be analyzed more in detail and described through vignettes from the interviews and excerpts from the descriptions of the infant's and mother's behaviors and the motherinfant interactions, during singing and non-singing episodes.

\section{Empowerment of the infant}

According to Haslbeck's definition (2013, 2014), empowerment of the infant refers to the infant's ability to relax, calm down, and enhance self-regulation, orientation, and interaction. This theme involves the infant's relaxation, the presentation of new competences, the participation and the engagement in singing, and for the purposes of this study, we also included the stabilization of oxygen saturation.

In the first MUSIP sessions, the infant was still unstable, showing frequent agitation and saturation drops. Therefore, in session four the empowerment of the infant could not be identified during singing and non-singing. However, in session six, Ana was more relaxed and in quiet sleep state during both singing and non-singing. Moreover, in session nine, singing contributed to a greater relaxation of the infant when compared to the non-singing episode. In this session the infant showed new competences, as well as participation and engagement during singing. In fact, while her mother was singing, the infant was in an alert quiet state, moving herself and vocalizing:

- $(05: 06)^{9}$ The mother adopts kangaroo care with the infant and she moves her closer to her face, while singing.

- (05:12) Ana vocalizes a glissando (La\#4 Re\#4 Mi4). The mother looks at the daughter and keeps singing.

- (05:28) Ana moves her head and vocalizes again (glissando from La4 to Mi4 approximately) and keeps moving her body. (S9/singing) ${ }^{10}$

In the post-MUSIP and pre-discharge period, the empowerment of the infant appeared more evident. For example, during the assessment interview of the intervention, Natalia reported that the MUSIP sessions and her spontaneous experiences of singing helped her daughter calm down when she was agitated,

But then, I realized that she liked me singing to her, with her in my arms or with her in the incubator, and touching her, I felt that it made her much calmer when she was agitated, she really liked it, even now she likes it. (Int3).

Besides enhancing the infant's relaxation, the MUSIP contributed to stabilizing Ana's oxygen saturation since the mother reported that when she sang, the saturation increased and kept stable. Natalia emphasized the joint positive effect of singing and kangaroo care on re-stabilizing oxygen saturation: "When her saturation starts dropping, I ask to pick her up, I sing and I rock her, and then she stabilizes" (Int3). Moreover, the mother reported that when she talked to Ana, her saturation kept decreasing, while when she sang the saturation remained more stable:

When I don't sing, she stays calm in her corner, then her saturation drops. But when I sing, when I hug her, and I sing to her, she stabilizes. Then she stays stable, it doesn't drop as much when I sing to her. I can see this. (Int4) 
Relaxation during maternal singing was also reported in the descriptions of the observation sessions in the post-MUSIP. In fact, the infant slept throughout the session, without displaying any signs of agitation, while the mother hummed improvised tunes, rocked, and caressed her. The mother also reported that after the music therapy sessions, the infant seemed to recognize the maternal voice and react to singing, by opening her eyes and smiling:

I feel that [the sessions] helped her, because she pays more attention to the voices, she already recognizes the voices, she already recognizes mine from the others ... from the nurses' voices, I think that she recognizes it; she already recognizes my voice. Sometimes, she opens her eyes, sometimes not [...], just gives a little smile at the corner of her mouth. (Int3).

In the pre- and post-discharge period, both the interview and the descriptions of the observation sessions highlighted the empowerment of the infant through the presentation of new competences and the engagement in singing, more than through relaxation as it was shown in the post-MUSIP. In fact, during singing and non-singing the infant interacted actively through her gaze, prolonged face-to-face contact with her mother, and vocalizations:

- (02:43) The infant looks at her mother.

- $(04: 34)$ The infant looks at her mother again and stops breastfeeding.

- $(04: 36)$ The mother stares at her daughter's face, looking at her, the infant follows her mother's gaze and they look at each other in a mutual gaze until 04:41 when the mother asks "What's the matter?".

- (05:02) The infant stops breastfeeding, follows her mother's gaze and moves her hand on her breast until 05:12.

- (05:36) The infant stretches her arm. (Ob4/non-singing);

- $(00: 45)$ The infant vocalizes in a more prolonged manner

- (00:59) The infant quietly moans and vocalizes again (Sol\#4 Fa\#4 Sol\#4), while the mother is dressing her. (Ob5/non-singing)

During singing there were more prolonged mother-infant gazes and also wider movements of the arms, diversified vocalizations, and several facial expressions. The infant looked at the mother more frequently when she was singing compared to the nonsinging episodes:

- $06: 41)$ During the break in maternal singing, the infant vocalizes in a clear and prolonged manner.

- (06:50) After moaning, the infant looks at the mother and moves her arms, while the mother keeps rocking her and singing.

- (07:30) Face-to-face contact and mother-infant gaze. Ana shows her tongue, Natalia smiles and sings.

- (07:33) Face-to-face contact, the mother smiles, the infant looks at another direction.

- (07: 38) The infant looks at the mother again, face-to-face contact, the mother smiles.

- (07:42) The mother keeps singing, rocking and looking at the daughter, the infant looks at her mother, face-to-face contact.

- $07: 45)$ The infant shows her tongue and at the same time the mother rubs the infant's nose with her own nose (face-to-face contact ends). (Ob5/singing)

\section{Empowerment of the mother}

According to Haslbeck's definition (2013, 2014), the empowerment of the mother refers to her ability to relax, calm down, interact with the infant, and increase maternal competences. This theme involves mother's relaxation, the strengthening of maternal competences, and in this study, it also includes the overcoming of the embarrassment and the fear of interacting with the infant and the autonomy in singing. 
During the first MUSIP sessions, the mother was anxious and agitated when she sang to her daughter. However, in session four she prepared herself for the singing experience by closing her eyes and leaning on the incubator, which was identified as an attempt to relax. The descriptions of session four showed the mother's autonomy in singing, since she hummed for her daughter without waiting for the music therapist:

- (11:52) The mother starts singing spontaneously in Sib major, the music therapist accompanies her.

- (12:16) Natalia starts the verse autonomously, by singing with open eyes and checking the infant's responses. (S4/singing)

The mother's relaxation was more evident in the descriptions of singing episodes related to sessions six and nine. While she was singing during kangaroo care with the infant, Natalia closed her eyes and leaned her head or mouth on Ana's head for a longer period. This behavior was not present in the non-singing episodes of the MUSIP, suggesting that Natalia probably associated this position with the experience of singing to her daughter. Furthermore, in these sessions, she overcame the embarrassment and the fear of interacting with her daughter, which have been reported as the mother's initial difficulties in the first interviews. She also showed maternal competences, by touching her daughter, caressing her, and being responsive to the infant's behaviors:

- (15:43) Natalia puts her mouth on Ana's head, caresses her body and hums very softly. The mother sings with closed eyes and caresses rhythmically her daughter's body. Natalia stays in the same position until 17:46. (S6/singing);

- (05:04) Ana moans and the mother looks at her, with a worried expression.

- (05:12) Ana vocalizes a glissando (La\#4 Re\#4 Mi4) and Natalia answers by kissing her and speaking to her gently. (S9/singing)

In the non-singing episodes of the same sessions (six and nine), the strengthening of maternal competences was also perceived. The mother touched her infant and used expressions of affection, but she altered her gaze focus more frequently. Sometimes, she looked at her daughter, while other times she looked at the NICU:

- (02:16) Natalia looks to the side.

- (02:19) The mother looks at her daughter.

- (02:32) The mother looks to the side and then she looks at her daughter again.

- (02:33) The mother says 'sh' to her daughter and kisses her head.

- (02:40) Natalia looks quickly in front of her and then again at her daughter.

- (02:44) The mother caresses her daughter's body and looks at her body. (S9/nonsinging)

Lastly, we observed greater autonomy of the mother in singing, compared to the prior sessions, since Natalia hummed improvised tunes to her daughter:

- (18:01) The mother stops caressing her daughter and sings without the accompaniment of the music therapist.

- (18:10) The mother improvised a melody, by humming autonomously. (S6/ singing)

In the post-MUSIP, the empowerment of the mother through relaxation appeared both in the interview and in the observation session. In fact, the mother reported that she "felt good" and more "relieved" during music therapy and when she sang to Ana. She highlighted that music therapy helped her to "loosen up," "feel less embarrassed" of talking to her daughter, and overcome the fear of touching her, since singing was always associated to touching or kangaroo care. This contributed to "build a bonding" between the mother and the infant, to stimulate Natalia's interest for her daughter and to help her "taking care" of Ana:

[The sessions] helped me a lot. In all, all senses. They helped me a lot with Ana, they helped me from A to Z. Because at the beginning I didn't care a lot, I didn't feel very close. But not now, now I want to know, I want to know why, I want to touch her, I want to pick her up, I want to move her, I want to interact with Ana. Before not so much, I was 
more... Not now, now I want to, I want to. And this helped me a lot, the sessions helped a lot (Int3).

Furthermore, music therapy contributed to the empowerment of maternal competences and the mother's autonomy in singing, since Natalia integrated the improvised humming in her daily routine at the NICU, by using singing as a resource to calm her daughter down when she was agitated or when the oxygen saturation dropped:

Once a day I always do it here [sing]. Even if for just a little while, but ...; She is calm in the incubator, but her saturation commonly drops. So I try to sing, I hug her and I sing to her, rocking her (Int3).

Similarly, the descriptions of the observation sessions showed that Natalia sang autonomously to her daughter, with improvised humming, and adding many prolonged and diversified expressions of affection:

- (02:21) Natalia opens her eyes, looks at her daughter and kisses her.

- (02:33) The mother looks at the daughter, takes her hand and gently pats her bum.

- (02:46) The mother touches her daughter's ear and cheek and, at the same time, she caresses Ana's head with her own cheek.

- (03:06) Natalia sings again, while she caresses her daughter and looks at her. (Ob3/singing)

In the pre-discharge interview (Phase 4), the empowerment of the mother was more visible through the strengthening of her competences and her autonomy in singing, than through relaxation. In fact, in this phase the interactive potential of singing was more evident. Whilst answering a question made by the interviewer about the contributions of the music therapy, Natalia reported:

Moreover, [the sessions helped me] to talk to her, I don't know, with everything. To feel closer to her, to loosen up, to caress her a little more, not to have so much fear, concern about ... staying close to her and talking to her, thinking that she didn't understand, but she understands everything, (Int4).

Despite this, in the pre-discharge period Natalia reported that she did not sing anymore for her daughter, because Ana was no longer agitated, she could pick her up more often, thus preferring to just talk to her.

In fact, in the pre- and post-discharge period, in the non-singing episodes, the mother talked to her daughter through infant-directed speech ${ }^{11}$ that is contingent with the infant's behaviors, such as gaze and sucking:

- (04:41) Mother-infant face-to-face contact, the infant does not suckle anymore and the mother repeats "What's the matter?".

- (04:48) The mother requests an answer from her daughter by saying "Mmm?" (Fa\#4) and rocks her gently, the infant looks at her mother.

- $(04: 51)$ Mother-infant face-to-face contact, the infant does not suckle, the mother asks "Don't you want to breastfeed anymore?".

- $(04: 54)$ The infant suckles again and the mother says "Ah! Yes, of course you want to!" while she takes her daughter's hand and caresses her face. (Ob4/non-singing).

Even though in the pre-discharge period the mother stopped singing for her daughter, she reported that she wanted to sing at home after discharge with the whole family when the infant would be agitated to calm her down and help her to sleep. According to Natalia, music therapy seemed to represent a transitional stage that helped her to awaken her maternal competences, to connect with the infant, and talk more to her. In the post-discharge (Phase 5) period, we observed that when Natalia sang she dedicated more exclusive attention to her daughter, showing more diversified expressions of affection and allowing Ana to more face-to-face contact and interactions:

- (08:05) Mother-infant face-to-face contact, the mother keeps patting her bum and singing, the infant looks at the mother. 
- (08:07) The mother caresses her daughter's nose with her own nose and at the same time the infant moans.

- $(08: 13)$ The infant shows her tongue, the mother smiles, takes her daughter's arms and moves them. (Ob5/singing)

In the pre- and post-discharge period we observed that the mother was responsive to her daughter's behaviors, for example imitating her facial expressions, answering to the infant's visual contact and stopping singing to wait for her daughter's answers:

(00:07) The infant makes visual contact with the mother and the mother says "Shush" to her. The infant looks at her.

- $(02: 45)$ The mother smiles, opens her eyes wider and turns her head to the right, then she caresses her daughter's face, head and body, mother-infant face-to-face contact. (Ob4/non-singing).

Nevertheless, during non-singing episodes the mother alternated her gaze focus, sometimes looking at her daughter and other times at the NICU environment, as previously pointed out:

- (03:28) The infant moves her eyes and stops breastfeeding. The mother looks at her infant, moving.

- (03:36) The infant starts breastfeeding again; the mother looks at her without moving.

- $03: 55)$ The mother looks in front of her, the infant keeps breastfeeding.

- (04:04) The mother looks at her daughter again, the infant breastfeeds and looks at her mother.

- (04:06) The mother looks again in front of her, the infant breastfeeds. (Ob4/nonsinging)

On the contrary, when the mother sang, the expressions of affection and face-to-face contact were more prolonged. In fact, after discharge, the mother looked at the infant during the whole observation session:

- $(06: 58)$ The infant looks to the side and then again at the mother and burps.

- (07:03) The mother smiles and caresses her nose with her own nose.

- (07:19) The infant stares at the mother with her eyes wide open.

- (07:22) Natalia imitates her expression.

- (09:22) The mother stops rocking her, stops singing and during the break she caresses her daughter's nose. Then she waits for the infant's answers, she caresses her and sings again. Face-to-face contact. (Ob5/singing).

\section{Discussion}

This study investigated the contributions of music therapy to the mother-preterm infant dyad in a Brazilian NICU. Based on the literature, our initial expectation was that music therapy would contribute to help both the mother and the infant to relax, to sensitize the mother to the importance of singing in the NICU as a resource for interacting with the infant, and to enhance the quality of mother-infant interaction.

Regarding the first theme that was analyzed, the empowerment of the infant increased as she grew and her health stabilized. In fact, she could gradually relax more, stabilize, and maintain the oxygen saturation, also presenting small competences. We observed these aspects over the last MUSIP sessions, as well as in the post-MUSIP and in the pre- and post-discharge periods, when Ana displayed new competences, by engaging herself during singing. Obviously, this evolution was associated both to the infant's improvement and to her stabilization. Nevertheless, evidence suggested that music therapy also contributed to it, since the infant was more engaged in the interaction during maternal singing, with prolonged and diversified gestures, vocalizations, gazes, and facial expressions.

These results support the findings of previous studies that showed the benefits of live music on the physiological and behavioral responses of the preterm infant (Arnon 
et al., 2006; Ettenberger et al., 2014; Garunkstiene et al., 2014; Loewy et al., 2013). In particular, live music interventions with infant-contingent singing promoted the infant's self-regulation and development (Haslbeck, 2014; Malloch et al., 2012; Shoemark, 2011). Besides music therapy intervention being with live singing, the MUSIP involved the mother's singing. Interventions that use maternal voice showed positive effects both on the infant and the mother, since the maternal voice is a privileged source of stimulation for the fetus and the newborn, and can promote mother-infant bonding and affective communication (Arnon et al., 2014; Butler et al., 2014; Cevasco, 2008; Filippa et al., 2013).

We believe that music therapy's benefits added to other positive factors, such as the mother-infant skin-to-skin contact during kangaroo care, which was always associated to singing since session six. Arnon et al. (2014) compared maternal singing during kangaroo care to kangaroo care alone in a randomized study with 86 infants and their mothers. The results showed that mothers felt less anxious and infants showed better stability of the autonomic nervous system when the mothers were singing. In our study, we observed a combined effect of maternal singing and kangaroo care on relaxation, stabilization of oxygen saturation, and on the development of the infant's competences.

As far as the empowerment of the mother is concerned, she could relax more throughout the MUSIP and in the follow-up sessions, overcome the embarrassment and the fear of interacting with the infant and improve her maternal competences, both in singing and non-singing episodes. Nevertheless, when the mother sang, she could better engage with her infant with expressions of affection, touching her and showing more prolonged face-to-face contact. Research showed that music therapy can reduce maternal anxiety and stress (Ak et al., 2015; Arnon et al., 2014; Cevasco, 2008), promote her relaxation and help her develop maternal competences (Ettenberger et al., 2014). In fact, singing can help the mother feel less powerless about prematurity and participate in her infant's well-being, contributing to the mother-infant relationship (Cevasco, 2008). In particular, in this study, the intervention was important to awaken maternal competences that might have been affected by the infant's premature birth, by the mother's personal difficulties and by the traumatic experience of the hospitalization in the NICU. Therefore, music therapy interventions should be implemented in the beginning of the hospital stay, in order to meet the mother's and infant's early needs, as well as the demands of the mother-infant relationship, especially in the context of prematurity. It is also important to develop family-centered interventions, including both parents in music therapy, guiding and supporting them in the vocal interaction with the preterm infant (Edwards, 2011; Haslbeck, 2014; Shoemark, 2011).

Altogether, our findings support previous research, showing that the MUSIP had positive effects on the infant, the mother, and their interaction. The mother's participation through singing provided her with the opportunity of actively engaging herself in her infant's care, reducing her feelings of incompetence in the NICU (Cevasco, 2008; Ettenberger et al., 2014). In particular, besides including the mother, the MUSIP provided a constant accompaniment of the dyad and valued the mother's musical preferences. Both factors were shown to be important for the efficacy of the intervention and the continued participation of mothers in these studies (Blumenfeld \& Eisenfeld, 2006; Ettenberger et al., 2014; Loewy, 2015). Both the mothers and the fathers of hospitalized infants in NICUs struggle with very challenging and intensive feelings. Therefore, it is important to provide them with longer interventions in order to establish a therapeutic relationship between the music therapist and the parents (Ettenberger et al., 2014). In our study, the role of the music therapist with her sensitive listening, was fundamental in encouraging maternal singing and in supporting the mother to feel more confident about her own competences and to engage more in the interaction with her daughter. The therapist's singing and guitar accompaniment were used as a resource of interaction with the mother-infant dyad, which contributed to establish the therapeutic relationship. However, studies with these characteristics are not so com- 
mon, since most research involves short and specific interventions, rarely addressed to the dyad and generally to the infant (Palazzi, Nunes, \& Piccinini, 2017).

The singing episodes highlighted the contingency between the mother's and infant's behaviors, showing that their empowerment contributed to their relationship. Some studies suggested that music-based interventions promote attachment (Cevasco, 2008), parental responsiveness (Walworth, 2007), mother-infant relationship (Ettenberger et al., 2014), and interactional synchrony (Haslbeck, 2014). For example, in the research conducted by Ettenberger et al. (2014), the mothers reported that music therapy helped them to be more motivated to stay with their infants, to know them better and to communicate more love to them. Similarly, in our study the mother reported that initially she was not very interested in her daughter, but music therapy helped her to be more motivated and to be willing to know, touch, stay and interact with her. During maternal singing, both the mother and the infant engaged more, shared more prolonged face-to-face contact and mutual gazes, and interacted with facial expressions, maternal voice, and infant's vocalizations. In fact, maternal singing has shown to provide a greater engagement of the infant and to increase attention, contributing to enhance the emotional coordination of the dyad (Nakata \& Trehub, 2004; Peretz, 2010).

Our results support the initial expectation that music therapy contributes to relax both the mother and the infant, to sensitize the mother to the importance of singing in the NICU, and to enhance the quality of mother-infant interaction. However, we also found unexpected results. For example, we highlight the mother's difficulties to sing to her daughter during the first four sessions, as well as her agitation and anxiety. This shows the importance of the presence of a music therapist in the NICU threatening environment to support and accompany maternal singing. The music therapist in this context can play the role of a mediator in the transition to motherhood, by helping the mother to awaken her maternal competences and facilitating mother-infant interaction.

\section{Limitations}

Before concluding, it is worth mentioning some limitations of this study. First, it was impossible to fully meet all the initial eligibility criteria, due to the complexity of the clinical conditions associated with prematurity. For example, the mother was not primipara and was already used to singing to her children. This might have contributed to strengthen the benefits of the intervention. On the other hand, she did not live with the father of the infant, had a limited availability of time, was worried about her children at home, and had little family support. All these factors might have made her engagement in the intervention more difficult. In addition, we have evidence from just one dyad, so it is important to adopt the MUSIP with a bigger sample.

Secondly, we could not investigate maternal anxiety and depression in the pre- and post- intervention, and we suggest to examine it in future studies. The presence of maternal depression might have complicated the intervention, by limiting the mother's engagement in singing to her daughter and in the interaction with her.

Thirdly, we adapted the application of the MUSIP, due to several conditions involving the infant, the mother, and the NICU, and we carried out just one individual session with the mother and all the other sessions with the mother-infant dyad. Despite being a limitation, the adaptation of the MUSIP also represented an advantage that made a more individualized intervention possible, suited to the needs of the motherinfant dyad. This might have contributed to strengthen the role of the mother in the interaction with her daughter. In fact, it is important to carry out interventions that are close to the clinical reality (Ettenberger et al., 2014) and, therefore, the application of the MUSIP requires the music therapist's sensitivity to the needs of the infant, the mother, and the NICU. Furthermore, it is important to highlight that this study was carried out in a public hospital in Porto Alegre, where the majority of the families of the hospitalized infants have a low income. Families in this socioeconomic situation 
may have more difficulties in getting involved in interventions. For instance, they may have problems in coming everyday to the hospital, because of the distance and costs of the travel. Mothers may have other children at home and may not have adequate social support for them while at the hospital. All this factors, together with personal difficulties to deal with the infant's premature birth, might have affected the implementation of the original protocol of the MUSIP.

Fourthly, the structure of the themes used in the analysis (Haslbeck, 2013, 2014) was originally based on the music therapist-infant interaction and was adapted for this study to analyze the mother-infant interaction. The fact that the analysis was based on a deductive approach from two of Haslbeck's themes might have contributed to disregard some aspects of our findings. Nevertheless, we tried to limit this risk, by adding to the themes some specific aspects emerging from the data of our study.

A qualitative approach was adopted to assess these themes, which allowed us to conduct an in-depth analysis, but it did not provide statistical data about the frequency of the observed behaviors. Lastly, the single case study does not provide an exhaustive comprehension of the phenomenon and the evolution of the mother-infant dyad was also due to factors such as the infant's development.

\section{Conclusions}

Despite these limitations, we might also highlight some methodological contributions of our study. The use of a qualitative approach and the emphasis on maternal perspective and the mother-infant interaction; all mentioned as rare aspects in research (Haslbeck, 2012).as well as data triangulation, through the interviews, to understand maternal perceptions and the direct observation of the mother-infant interaction. The intervention we proposed also has relevant contributions. It included the participation of the dyad, it provided emotional support for the mother, and the opportunity to develop new abilities through singing to her preterm daughter; it contributed to the empowerment of the dyad and to humanize and "harmonize" the threatening environment of the NICU (Cleveland, 2008). In conclusion, the intervention also may have had a multiplier effect on other mothers who did not directly participate in the MUSIP, but whose awareness about the importance of singing to their hospitalized infants was raised.

This study supports the literature, by showing the contributions of music therapy to the preterm infant, the mother and the mother-infant interaction. Music and maternal singing, being universal and early elements in mother-infant bonding, allowed the mother to approach her daughter, even in the adverse environment of the NICU.

Music therapy also contributed to the psychological well-being of this mother and to her relationship with her preterm daughter. Therefore, we stress the importance of including music therapy in NICU care, since it is a cost-effective intervention that promotes the infant's development, the maternal well-being, and the mother-infant bonding. Investing in the mother-infant relationship is fundamental both to infant's development and to the public health, since it contributes to health promotion and prevention and it decreases the expensive costs commonly involved in prematurity.

In the light of the evidence reported in this study, new studies will be necessary to examine MUSIP potential and its effectiveness. New studies with a longitudinal design are needed in order to investigate the long-term effects of music therapy on the mother and her infant. Lastly, it is fundamental to involve in the interventions not just the mother, but also the father, focusing on the dyad and on the triad interaction, and investigating the parental perspectives about music therapy in the context of prematurity.

\section{Notes}

1. All names were altered to preserve the privacy of the participants. 
2. The calculated obstetric gestational age was of 23 weeks, while Ballard score indicated 27 weeks. In neonatology, the Ballard assessment is a commonly used technique to calculate a newborn's gestational age. It assigns a score to both neurologic and physical characteristics. The sum of all criteria allows an estimate of gestational ages between 26 and 44 weeks. The New Ballard Score adds other criteria, which determine gestational ages prior to 20 weeks (Ballard et al., 1991).

3. The Apgar score assesses the clinical status of the newborn infant, according to five components (heart rate, respiration, muscle tone, reflex irritability and skin color) at the 1st, 5th, and 10th minute after birth, and assigns a range from 0 to 10 to each component. The Apgar score, which is the result of the sum of the five components, allows the classification of the newborn without asphyxia (Apgar score of 8 to 10), with mild asphyxia (Apgar score of 5 to 7), with moderate asphyxia (Apgar score of 3 to 4), and with severe asphyxia (Apgar score of 0 to 2) (Apgar, 1953).

4. Post-menstrual age is considered as the sum of the gestational age at birth (time elapsed between the first day of the last normal menstrual period and the day of delivery) and the chronological age (time elapsed after the infant's birth) (AAP, 2004).

5. A more detailed description of the MUSIP original protocol can be found in the first author's Master's dissertation (Palazzi, 2016).

6. Session four was selected for being the first videotaped session with the mother-infant dyad; session six was chosen for being the first session with the mother and the infant during kangaroo care, and session nine was selected for being the last session of the intervention.

7. In session six, the first non-singing episode was much shorter than the last singing episode. Therefore, we selected the first two non-singing episodes after the selected singing episode in the same session.

8. In the observation session during diaper change, carried out in Phase 5, the video excerpt lasted less than 3 minutes; therefore the whole video was selected for the analysis.

9. These time indications refer to the time identified in the analysis of MUSIP video footage and of the observation sessions.

10. The letter 'S', followed by a number, refers to the MUSIP session (session four, six, or nine); the abbreviations 'Int' and 'Ob' refer respectively to the maternal speech from the interviews or the descriptions derived from the sessions of observation; lastly, the number that follows the abbreviations 'Int' and 'Ob' refers to the phase in which the interviews or sessions took place (Phase 3, 4, or 5).

11. The expressions "infant directed-speech", "motherese," or "baby-talk" refer to the prototypical speech that parents and caregivers use when they talk to infants or children. Infant-directed speech has a higher pitch and is warmer, more rhythmic, repetitive, and contingent with the infant's state (Saint-Georges et al., 2013).

\section{References}

Ak, J., Lakshmanagowda, P. B., G C M, P., \& Goturu, J. (2015). Impact of music therapy on breast milk secretion in mothers of premature newborns. Journal of Clinical and Diagnostic Research, 9(4), CC04-CC06, https://doi.org/http://dx.doi.org/10.7860/JCDR/2015/ 11642.5776.

Aldridge, D., \& Aldridge, G. (2002, 02 12). Music Therapy Today (online). Therapeutic narrative analysis: A methodological proposal for the interpretation of music therapy traces., Retrieved from, http://musictherapyworld.net/modules/mmmagazine/issues/ 20021205144406/20021205150303/TherapeutNarrativeAnalysis.pdf.

Alipour, Z., Eskandari, N., Ahmari Tehran, H., Eshagh Hossaini, S. K., \& Sangi, S. (2013). Effects of music on physiological and behavioral responses of premature infants: A randomized controlled trial. Complementary Therapies in Clinical Practice, 19(3), 128-132, https://doi.org/10.1016/j.ctcp.2013.02.007. 
Al-Qahtani, N. H. (2005). Foetal response to music and voice. The Australian and New Zealand Journal of Obstetrics and Gynaecology, 45(5), 414-417, https://doi.org/10.1111/ j.1479-828X.2005.00458.x.

Apgar, V. (1953). A Proposal for a New Method of Evaluation of the Newborn Infant. Anesthesia \& Analgesia, 32(1), 260-267, https://doi.org/10.1213/ 00000539-195301000-00041.

Arnon, S., Shapsa, A., Forman, L., Regev, R., Bauer, S., Litmanovitz, I., \& Dolfin, T. (2006). Live Music Is Beneficial to Preterm Infants in the Neonatal Intensive Care Unit Environment. Birth, 33(2), 131-136, https://doi.org/10.1111/j.0730-7659.2006.00090.x.

Arnon, S., Diamant, C., Bauer, S., Regev, R., Sirota, G., \& Litmanovitz, I. (2014). Maternal singing during kangaroo care led to autonomic stability in preterm infants and reduced maternal anxiety. Acta Paediatrica, 103, 1039-1044, http://dx.doi.org/10.1111/apa.12744.

American Academy of Pediatrics. (2004). Age Terminology During the Perinatal Period. Pediatrics, 114, 1362. Retrived from: http://pediatrics.aappublications.org/content/114/5/ 1362.full.html

Ballard, J. L., Khoury, J. C., Wedig, K., Wang, L., Eilers-Walsman, B. L., \& Lipp, R. (1991). New Ballard Score, expanded to include extremely premature infants. The Journal of Pediatrics, 119(3), 417-423, https://doi.org/10.1016/S0022-3476(05)82056-6.

Bieleninik, Ł., Ghetti, C., \& Gold, C. (2016). Music therapy for preterm infants and their parents: A meta-analysis. Pediatrics, 138(3), e20160971, http://dx.doi.org/10.1542/ peds.2016-971.

Bilgin, A., \& Wolke, D. (2015). Maternal sensitivity in parenting preterm children: A metaanalysis. Pediatrics, 136(1), e177-e193, http://dx.doi.org/10.1542/peds.2014-3570.

Blumenfeld, H., \& Eisenfeld, L. (2006). Does a mother singing to her premature baby affect feeding in the neonatal intensive care unit? Clinical Pediatrics, 45(1), 65-70, http://dx.doi.org/10.1177/000992280604500110.

Braun, V., \& Clarke, V. (2006). Using thematic analysis in psychology. Qualitative Research in Psychology, 3(2), 77-101, http://dx.doi.org/10.1191/1478088706qp063oa.

Butler, S. C., O’Sullivan, L. P., Shah, B. L., \& Berthier, N. E. (2014). Preference for infantdirected speech in preterm infants. Infant Behavior \& Development, 37, 505-511, http://dx.doi.org/10.1016/j.infbeh.2014.06.007.

Cevasco, A. M. (2008). The effects of mothers' singing on full-term and preterm infants and maternal emotional responses. Journal of Music Therapy, 45(3), 273-306, http://dx.doi.org/ 10.1093/jmt/45.3.273.

Cleveland, L. M. (2008). Parenting in the neonatal intensive care unit. JOGNN, 37, 666-691, http://dx.doi.org/10.1111/j.1552-6909.2008.00288.x.

Edwards, J. (2011). The use of music therapy to promote attachment between parents and infants. The Arts in Psychotherapy, 38, 190-195, http://dx.doi.org/10.1016/ j.aip.2011.05.002.

Ettenberger, M., Odell-Miller, H., Cárdenas, C. R., Serrano, S. T., Parker, M., \& Llanos, S. M. C. (2014). Music therapy with premature infants and their caregivers in Colombia - A mixed methods pilot study including a randomized trial. Voices: A World Forum for Music Therapy, 14(2), https://voices.no/index.php/voices/article/view/756>.

Filippa, M., Devouche, E., Arioni, C., Imberty, M., \& Gratier, M. (2013). Live maternal speech and singing have beneficial effects on hospitalized preterm infants. Foundation Acta Pcediatrica, 102(10), 1017-1020, http://dx.doi.org/10.1111/apa.12356.

Flacking, R., Ewald, U., Nyqvist, K. H., \& Starrin, B. (2006). Trustful bonds: a key to "becoming a mother" and to reciprocal breastfeeding. Stories of mothers of very preterm infants at a neonatal unit. Social Science \& Medicine, 62(1), 70-80, http://dx.doi.org/ 10.1016/j.socscimed.2005.05.026. 
Fleck, A., \& Piccinini, C. A. (2013). O bebê imaginário e o bebê real no contexto da prematuridade: do nascimento ao $3^{\circ}$ mês após a alta. [The imaginary baby and the real baby in the context of prematurity: from birth to 3 months after discharge]. Aletheia, 40, 14-30.

Forcada-Guex, M., Pierrehumbert, B., Borghini, A., Moessinger, A., \& Muller-Nix, C. (2006). Early dyadic patterns of mother-infant interactions and outcomes of prematurity at 18 months. Pediatrics, 118(1), e107-114, http://dx.doi.org/10.1542/peds.2005-1145.

Garunkstiene, R., Buinauskiene, J., Uloziene, I., \& Markuniene, E. (2014). Controlled trial of live versus recorded lullabies in preterm infants. Nordic Journal of Music Therapy, 23, 71-88, http://dx.doi.org/10.1080/08098131.2013.809783.

Glaser, B., \& Strauss, A. (1967). The discovery of grounded theory. New York, NY: Aldine.

Haslbeck, F. B. (2012). Music therapy for premature infants and their parents: An integrative review. Nordic Journal of Music Therapy, 21, 203-226, http://dx.doi.org/10.1080/ 08098131.2011.648653.

Haslbeck, F. B. (2013). Creative music therapy with premature infants: An analysis of video footage. Nordic Journal of Music Therapy, 23(1), 5-35, https://doi.org/10.1080/ 08098131.2013.780091.

Haslbeck, F. B. (2014). The interactive potential of creative music therapy with premature infants and their parents: A qualitative analysis. Nordic Journal of Music Therapy, 23(1), 36-70, http://dx.doi.org/10.1080/08098131.2013.790918.

Keidar, R. H., Mandel, D., Mimouni, F. B., \& Lubetzky, R. (2014). Bach music in preterm infants: No "Mozart effect" on resting energy expenditure. Journal of Perinatology, 34(2), 153-155, http://dx.doi.org/10.1038/jp.2013.138.

Keith, D. R., Weaver, B. S., \& Vogel, R. L. (2012). The effect of music-based listening interventions on the volume, fat content, and caloric content of breast milk-produced by mothers of premature and critically ill infants. Advances in Neonatal Care, 12(2), 112-119, http://dx.doi.org/10.1097/ANC.0b013e31824d9842.

Kisilevsky, B. S., Hains, S. M. J., Lee, K., Xie, X., Huang, H., Ye, H. H., Zhang, K., \& Wang, Z. (2003). Effects of experience on fetal voice recognition. Psychological Science, 14(3), 220-224, http://dx.doi.org/10.1111/1467-9280.02435.

Korja, R., Latva, R., \& Lehtonen, L. (2012). The effects of preterm birth on mother-infant interaction and attachment during the infant's first two years. Acta Obstetricia et Gynecologica Scandinavica, 91, 164-173, http://dx.doi.org/10.1111/ j.1600-0412.2011.01304.x.

Linhares, M. B. M., Carvalho, A. E. V., Machado, C., \& Martinez, F. E. (2003). Desenvolvimento de bebês nascidos pré-termo no primeiro ano de vida. [Development of premature infants in the first year of life]. Cadernos de Psicologia e Educação - Paidéia, 1, 57-72.

Loewy, J., Stewart, K., Dassler, A. M., Telsey, A., \& Homel, P. (2013). The effects of music therapy on vital signs, feeding, and sleep in premature infants. Pediatrics, 131(5), 902-918, http://dx.doi.org/10.1542/peds.2012-1367.

Loewy, J. (2015). NICU music therapy: Song of kin as critical lullaby in research and practice. Annals of the New York Academy of Sciences, 1337, 178-185, http://dx.doi.org/10.1111/ nyas. 12648 .

Malloch, S., \& Trevarthen, C. (Eds.). (2009). Communicative musicality. Exploring the basis of human companionship. England: Oxford University Press.

Malloch, S., Shoemark, H., Črnčec, R. N. C., Paul, C., Prior, M., \& Coward, S. (2012). Music therapy with hospitalized infants - The art and science of communicative musicality. Infant Mental Health Journal, 33(4), 386-399, http://dx.doi.org/10.1002/imhj.21346.

March of Dimes, PMNCH, Save the Children, \& WHO. (2012). In C. P. Howson, M. V. Kinney, \& J. E. Lawn (Eds.), Born Too Soon: The Global Action Report on Preterm Birth. World Health Organization: Geneva. 
McMahon, E., Wintermark, P., \& Lahav, A. (2012). Auditory brain development in premature infants: the importance of early experience. Annals of the New York Academy of Sciences, 1252, 17-24, http://dx.doi.org/10.1111/j.1749-6632.2012.06445.x.

Moon, C. M., \& Fifer, W. P. (2000). Evidence of transnatal auditory learning. Journal of Perinatology, 20(8 Pt 2), 37-44.

Moreira, C. I., Gerhardt, C., Steibel, D., Silveira, F., Caron, N. A., \& Lopes, R. C. S. (2011). A impossível tarefa de segurar o sol com a mão. [The impossible task of holding the sun with your hand]. Revista de Psicanálise da SPPA, 18(2), 237-253.

Nakata, T., \& Trehub, S. E. (2004). Infants' responsiveness to maternal speech and singing. Infant Behavior \& Development, 27, 455-456, http://dx.doi.org/10.1016/ j.infbeh.2004.03.002.

Nöcker-Ribaupierre, M., Linderkamp, O., \& Riegel, K. P. (2015). The Effects of mothers' voice on the long term development of premature infants: A prospective randomized study. Music \& Medicine, 7(3), 20-25.

Nordoff, P., \& Robbins, C. (1977). Creative music therapy: Individualized treatment for the handicapped child. New York, NY: John Day.

Núcleo de Infância e Família - Federal University of Rio Grande do Sul - PREPAR Project. (2009a). Interview about motherhood in the context of prematurity. Unpublished instrument.

Núcleo de Infância e Família - Federal University of Rio Grande do Sul - PREPAR Project. (2009b). Demographic data sheet. Unpublished instrument.

Núcleo de Infância e Família - Federal University of Rio Grande do Sul - PREPAR Project. (2009c). Clinical data sheet for the mother and her preterm infant/post-partum. Unpublished instrument.

Núcleo de Infância e Família - Federal University of Rio Grande do Sul - PREPAR Project. (2014b). Assessment interview of the music therapy intervention for the mother and her preterm infant. Unpublished instrument.

Palazzi, A. (2016). Contributions of music therapy for the mother-preterm infant dyad in the NICU. Federal University of Rio Grande do Sul, Psychology Institute. Porto Alegre, Brazil. Master's Dissertation.

Palazzi, A., \& Piccinini, C. A. (2014). Observation of the mother-preterm infant interaction. Unpublished instrument.

Palazzi, A., Meschini, R., \& Piccinini, C. A. (2014a). Interview about the mother's musical history. Unpublished instrument.

Palazzi, A., Meschini, R., \& Piccinini, C. A. (2014b). Music Therapy Intervention for the MotherPreterm Infant Dyad - MUSIP. Unpublished instrument.

Palazzi, A., Nunes, C. C., \& Piccinini, C. A. (2017). Music therapy and musical stimulation in the context of prematurity: a review of literature from 2010 to 2015. Journal of Clinical Nursing, http://dx.doi.org/10.1111/jocn.13893.

Peretz, I. (2010). Towards a neurobiology of musical emotions. In P. Sloboda (Ed.), Handbook of Music and Emotion: Theory, research, applications (pp. 99-126). Oxford: Oxford University Press.

Saint-Georges, C., Chetouani, M., Cassel, R., Apicella, F., Mahdhaoui, A., Muratori, F., Laznik, M. C., \& Cohen, D. (2013). Motherese in interaction: at the cross-road of emotion and cognition? (A systematic review). PloS One, 8, e78103, 10.1371/journal.pone.0078103.

Shaw, R. J., Lilo, E. A., Storfer-Isser, A., Ball, M. B., Proud, M. S., Vierhaus, N. S., Huntsberry, A., Mitchell, K., Adams, M. M., \& Horwitz, S. M. (2014). Screening for Symptoms of Postpartum Traumatic Stress in a Sample of Mothers with Preterm Infants. Issues in Mental Health Nursing, 35(3), 198-207.

Shoemark, H. (2011). Translating 'infant-directed singing' into a strategy for the hospitalizaed family. In J. Edwards (Ed.), Music Therapy and Parent-Infant Bonding (pp. 161-178). New York: Oxford University Press.

Stake, R. E. (2006). Multiple case study analysis. New York, NY: Guilford Press. 
Standley, J. M. (2012). Music therapy research in the NICU: An updated meta-analysis. Neonatal Network: The Journal of Neonatal Nursing, 31(5), 311-316.

Standley, J., Cassidy, J., Grant, R., Cevasco, A., Szuch, C., Nguyen, J., Walworth, D., Procelli, D., Jarred, J., \& Adams, K. (2010). The effect of music reinforcement for non-nutritive sucking via the PAL (Pacifier-Activated Lullabies Apparatus) on achievement of oral feeding by premature infants in the NICU. Pediatric Nursing, 36(3), 138-145.

Strauss, A. L., \& Corbin, J. (1998). Grounded theory methodology. An overview. In N. Denzin \& Y. S. Lincoln (Eds.), Strategies of qualitative inquiry (pp. 158-183). London, England: Sage.

Trehub, S. E., Becker, J., \& Morley, I. (2015). Cross-cultural perspectives on music and musicality. Philosophical Transactions of the Royal Society B: Biological Sciences, 370(1664), 20140096, https://doi.org/10.1098/rstb.2014.0096.

Ullsten, A., Eriksson, M., Klässbo, M., \& Volgsten, U. (2016). Live music therapy with lullaby singing as affective support during painful procedures: A case study with microanalysis. Nordic Journal of Music Therapy, 26(2), 142-166, https://doi.org/10.1080/ 08098131.2015.1131187.

Vianna, M. N. S., Barbosa, A. P., Carvalhaes, A. S., \& Cunha, A. J. L. A. (2011). Music therapy may increase breastfeeding rates among mothers of premature newborns: a randomized controlled trial. Jornal de Pediatria, 87(3).

Walworth, D. D. (2007). The effect of developmental music groups for parents and premature or typical infants under two years on parental responsiveness and infant social development. The Florida State University. Electronic Theses, Treatises and Dissertations, Paper 1271.

World Health Organization. (2010). Bulletin of the World Health Organization, 88, 31-38. http://dx.doi.org/10.2471/BLT.08.062554

White-Traut, R., \& Norr, K. (2009). Ecological Model for Premature Infant Feeding. Journal of Obstetric, Gynecologic \& Neonatal Nursing, 38(4), 478-490, http://dx.doi.org/10.1111/ j.15526909.2009.01046.x. 